\title{
Cosmological Distances in Five General Cosmic Models
}

\author{
Fadel A. Bukhari \\ Department of Astronomy, Faculty of Science, King Abdulaziz University, Jeddah, Saudi Arabia \\ Email: flbukhari@hotmail.com
}

Received January 3, 2013; revised February 4, 2013; accepted February 12, 2013

Copyright (C) 2013 Fadel A. Bukhari. This is an open access article distributed under the Creative Commons Attribution License, which permits unrestricted use, distribution, and reproduction in any medium, provided the original work is properly cited.

\begin{abstract}
Four cosmological distances were investigated in the light of the five general cosmic models which were developed in a previous study. These are the proper distance, luminosity distance, angular diameter distance and distance modulus. Each of these distances was studied in terms of the redshift of the extragalactic objects. Estimations of the horizon distance of the universe, the total mass and the mass of matter within the horizon distance, the equivalent numbers of the Milky Way-like galaxies and the Coma-like clusters of galaxies to the mass of matter were determined in the general models at the present time.
\end{abstract}

Keywords: Extragalactic Distances; Cosmology; Cosmic Dynamics

\section{Introduction}

One of the most interesting aspects of investigating cosmic models is determination of cosmological distances of the extragalactic objects in these models such as the proper distance, luminosity distance, angular diameter distance (the proper distance of the object when its light was emitted) and distance modulus.

In a previous study five general cosmic models were developed using comprehensive selection ratios of the universe components which are luminous matter, dark matter, photons, neutrinos, and dark energy [1]. Although some physical properties of the universe were discussed in these models, we concentrate in this article on precise determination of cosmic distances of the extragalactic objects in terms of their redshifts.

The importance of this study comes from the fact that the cosmic distances mentioned above are most commonly used in many cosmological researches. As a consequence of specifying cosmic distances, interesting estimations were computed in the general models at the present time. These estimations are horizon distance, total mass within the horizon distance, mass of matter (luminous and dark matter) within the horizon distance, and its equivalent numbers of both the Milky Way-like galaxies and the Coma-like clusters.

Description of methodology is given in Section 2, while results and discussion are presented in Section 3. Accuracy and errors are illustrated in Section 4, and conclusions are shown in Section 5.

\section{Methodology}

The proper distance of an extragalactic object at present time $t_{0}$ is given by

$$
d_{p}\left(t_{0}\right)=R\left(t_{0}\right) f\left(r_{0}\right) .
$$

where $r_{0}$ is the radial comoving coordinate of the object now, and $f\left(r_{0}\right)$ is expressed as

$$
f\left(r_{0}\right)=c \int_{t_{e}}^{t_{0}} \frac{\mathrm{d} t}{R(t)}=\int_{0}^{r_{0}} \frac{\mathrm{d} r}{\sqrt{1-k r^{2}}}= \begin{cases}r_{0} & (k=0) \\ \sin ^{-1} r_{0} & (k=+1) . \\ \sinh ^{-1} r_{0} & (k=-1)\end{cases}
$$

where $R(t)$ is the scale factor of the universe expansion, $c$ the speed of light, $t_{e}$ the emission time of the object photon and $k$ the curvature of space in the universe. Let $a(t)=\frac{R(t)}{R\left(t_{0}\right)}$ then Equation (2) becomes

$$
f\left(r_{0}\right)=\frac{c}{R\left(t_{0}\right)} \int_{t_{e}}^{t_{0}} \frac{\mathrm{d} t}{a(t)} .
$$

Substituting by Equation (3) in Equation (1) we get

$$
d_{p}\left(t_{0}\right)=c \int_{t_{e}}^{t_{0}} \frac{\mathrm{d} t}{a(t)}
$$

From [1] we have seen that the expansion speed of the universe is 


$$
\dot{a}(t)=H_{0}\left[1-\Omega_{\Lambda, 0}\left(1-a^{2}\right)+\Omega_{m, 0}\left(\frac{1}{a}-1\right)+\Omega_{r, 0}\left(\frac{1}{a^{2}}-1\right)\right]^{1 / 2} .
$$

where $\Omega_{\Lambda, 0}, \Omega_{m, 0}$ and $\Omega_{r, 0}$ are the density parameters of the dark energy, matter and radiation at the present time respectively. $H_{0}$ is the Hubble parameter at the present time. Equation (5) can be written as

$$
\frac{\mathrm{d} t}{\mathrm{~d} a}=\frac{1}{H_{0}}\left[1-\Omega_{\Lambda, 0}\left(1-a^{2}\right)+\Omega_{m, 0}\left(\frac{1}{a}-1\right)+\Omega_{r, 0}\left(\frac{1}{a^{2}}-1\right)\right]^{-1 / 2} .
$$

Substituting by Equation (6) in Equation (4) we find

$$
d_{p}\left(t_{0}\right)=\frac{c}{H_{0}} \int_{(1+z)^{-1}}^{1} \frac{1}{a}\left[1-\Omega_{\Lambda, 0}\left(1-a^{2}\right)+\Omega_{m, 0}\left(\frac{1}{a}-1\right)+\Omega_{r, 0}\left(\frac{1}{a^{2}}-1\right)\right]^{-1 / 2} \mathrm{~d} a .
$$

where $1+z=\frac{1}{a\left(t_{e}\right)}, z$ is the redshift of the object.

The luminosity distance $d_{L}\left(t_{0}\right)$, angular diameter distance $d_{A}\left(t_{0}\right)$ and distance modulus of the object at the present time are expressed respectively as

$$
d_{L}\left(t_{0}\right)=d_{p}\left(t_{0}\right)(1+z) .
$$

$$
\begin{aligned}
& d_{A}\left(t_{0}\right)=\frac{d_{p}\left(t_{0}\right)}{1+z} . \\
& m-M=5 \log d_{L}+25 .
\end{aligned}
$$

where $m, M$ are the apparent and absolute magnitudes of the object. The distances $d_{p}, d_{L}$ and $d_{A}$ are measured in Mpc.

It is obvious from Equation (7) that the horizon distance of the universe at the present time is given by

$$
d_{h}\left(t_{0}\right)=\frac{c}{H_{0}} \int_{0}^{1} \frac{1}{a}\left[1-\Omega_{\Lambda, 0}\left(1-a^{2}\right)+\Omega_{m, 0}\left(\frac{1}{a}-1\right)+\Omega_{r, 0}\left(\frac{1}{a^{2}}-1\right)\right]^{-1 / 2} \mathrm{~d} a .
$$

where $z=\infty$. The Robertson-Walker metric of spacetime which describes the expansion or contraction of homogeneous and isotropic universe is given by

$$
d s^{2}=c^{2} d t^{2}-R^{2}(t)\left[\frac{d r^{2}}{1-k r^{2}}+r^{2} d \theta^{2}+r^{2} \sin ^{2} \theta d \phi^{2}\right] .
$$

where $r, \theta, \phi$ are the comoving coordinates of a point in space. The volume of three dimensional sphere can be written as

$$
V(t)=2 \int_{0}^{2 \pi} \mathrm{d} \phi \int_{0}^{\pi} \mathrm{d} \theta \int_{0}^{r_{0}} \sqrt{\operatorname{det} g_{R W}} \mathrm{~d} r .
$$

where $\operatorname{det} g_{R W}$ is the determinant of the spatial part of the Robertson-Walker metric matrix and given by

$$
\operatorname{det} g_{R W}=R^{6}(t) \frac{r^{4} \sin ^{2} \theta}{\sqrt{1-k r^{2}}} .
$$

Since the space of the universe is flat at the present time, hence $k=0$ and

$$
\sqrt{\operatorname{det} g_{R W}}=R^{3}\left(t_{0}\right) r^{2} \sin \theta
$$

Substituting by (15) in (13) we have

$$
V\left(t_{0}\right)=2 R^{3}\left(t_{0}\right) \int_{0}^{2 \pi} \mathrm{d} \phi \int_{0}^{\pi} \sin \theta \mathrm{d} \theta \int_{0}^{r_{0}} r^{2} \mathrm{~d} r .
$$

$$
V\left(t_{0}\right)=\frac{8 \pi}{3} R^{3}\left(t_{0}\right) r_{0}^{3} .
$$

For flat space (1) and (2) give

$$
d_{p}\left(t_{0}\right)=R\left(t_{0}\right) r_{0} .
$$

Substituting by (17) in (16) we get at the present time

$$
V\left(t_{0}\right)=\frac{8 \pi}{3} d_{p}^{3}\left(t_{0}\right) .
$$

Similarly the volume of sphere of radius $d_{h}\left(t_{0}\right)$ is expressed as

$$
V_{h}\left(t_{0}\right)=\frac{8 \pi}{3} d_{h}^{3}\left(t_{0}\right) .
$$

From the previous study [1] it is obvious that at any given cosmic time the total density of the universe is given by

$$
\begin{gathered}
\rho(t)=\rho_{m, t}+\frac{\rho_{r, t}}{c^{2}}+\frac{\rho_{\Lambda}}{c^{2}}, \\
\rho(t)=\Omega_{m, t} \rho_{c, t}+\Omega_{r, t} \frac{\epsilon_{c, t}}{c^{2}}+\Omega_{\Lambda, t} \frac{\epsilon_{c, t}}{c^{2}}, \\
\rho(t)=\rho_{c, t}\left(\Omega_{m, t}+\Omega_{r, t}+\Omega_{\Lambda, t}\right),
\end{gathered}
$$

or 


$$
\rho(t)=\rho_{c, t} \Omega(t) .
$$

where the parameters

$\rho_{m, t}, \rho_{r, t}, \rho_{\Lambda}, \Omega_{m, t}, \Omega_{r, t}, \Omega_{\Lambda, t}, \rho_{c, t}, \varepsilon_{c, t}$ and $\Omega(t)$ are all defined exactly as in the previous study. At the present time Equation (20) gives

$$
\rho\left(t_{0}\right)=\rho_{c, 0} \Omega_{0} .
$$

The total mass within $d_{h}\left(t_{0}\right)$ is given by Equations (19), (21) as

$$
M_{h}\left(t_{0}\right)=V_{h}\left(t_{0}\right) \rho\left(t_{0}\right) .
$$

The mass of matter within $d_{h}\left(t_{0}\right)$ is

$$
M_{m}\left(t_{0}\right)=M_{h}\left(t_{0}\right) \frac{\rho_{m, 0}}{\rho\left(t_{0}\right)},
$$

or

$$
M_{m}\left(t_{0}\right)=M_{h}\left(t_{0}\right) \frac{\Omega_{m, 0}}{\Omega_{0}} .
$$

Since the masses of the Milky Way galaxy $M_{M W}$ and the Coma cluster $M_{\text {СОMA }}$ are

$M_{M W}=8 \times 10^{11} \mathrm{M}_{\odot}, M_{\text {COMA }}=2 \times 10^{15} \mathrm{M}_{\odot},[2]$, where $\mathrm{M}_{\odot}$ is the solar mass. Therefore the equivalent numbers of the Milky Way-like galaxies and the Coma-like clusters to $M_{m}\left(t_{0}\right)$ are respectively

$$
\begin{gathered}
N_{M W}=\frac{M_{m}\left(t_{0}\right)}{M_{M W}} . \\
N_{\text {COMA }}=\frac{M_{m}\left(t_{0}\right)}{M_{\text {СOMA }}} .
\end{gathered}
$$

\section{Results and Discussion}

The proper distance-redshift relation (7) in the general models up to $z=6$ is plotted in Figure 1, where the distributions increase with $z$ and they coincide on each other for $z \leq 2.806$, but they slightly diverge from each other afterwards such that the distributions of the general models $A$ and $C$ are at the top followed by those of the general models $B, D$, and $E$ respectively.

The distributions of the luminosity distance-redshift relation in the general models up to $z=1$ are shown in Figures 2(a)-(e). In each figure the upper curve represents Equation (8) and the lower curve represents the relation

$$
d_{L}=\frac{c}{H_{0} q_{0}^{2}}\left[z q_{0}+\left(q_{0}-1\right)\left\{\left(2 q_{0} z+1\right)^{1 / 2}-1\right\}\right] .
$$

where $q_{0}$ is the decelerating parameter of the universe at the present time $[3,4]$. It is noticeable that $d_{L}$ continuously raises with $z$, however, Equation (26) gives less estimation of $d_{L}$ than that of Equation (8) for $z>0.15$ and the difference between the two estimations increases also with $z$. This is due to the repulsive effect of the dark energy. Initially, when the universe has small volume the effect of gravity is greater than the effect of dark energy, so the initial expansion rate of the universe is slow just as in Friedmaun models. However, there will come a point when the repulsive effect of the dark energy will equal and then exceeds the effect of gravity and the universe will expand in continuously increasing rate. In this case, distant galaxies will have greater distances from us than their distances in the case of the Friedmaun models [5].

Figure 3 illustrates the distributions of the angular diameter distance - redshift relation (9) in the general models up to $z=6$. These distributions coincide on each other for $z \leq 2.8182$, then they start diverging slightly from each other with the distributions of the general models $A, C$ and $B$ are at the top followed by those of the general models $D$ and $E$ respectively. All distributions raise up appreciably fast until the value $Z=z_{\max }$ given in each general model in Table 1, then the distributions decrease gradually with increasing $z$.

The distance modulus-redshift relation is determined using Equations (8) and (10) and represented by the

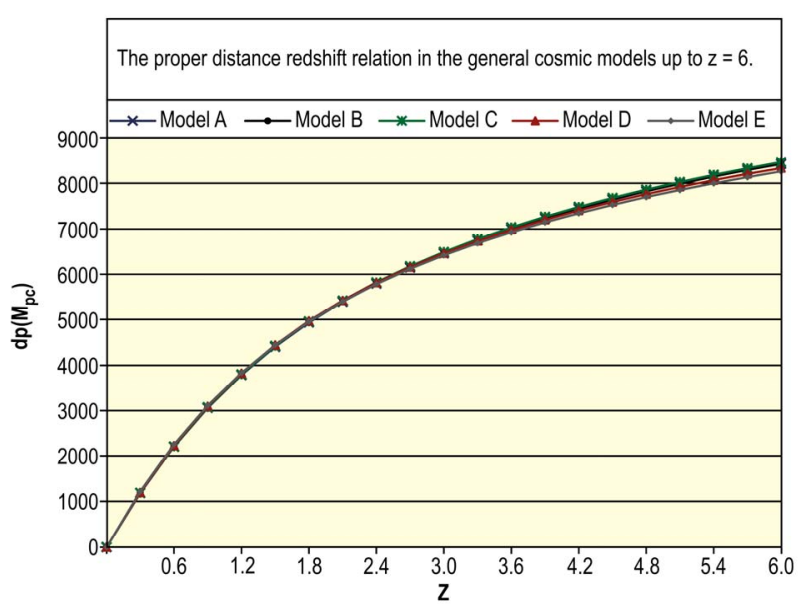

Figure 1. The proper distance redshift relation in the general cosmic models up to $z=6$.

Table 1. Maximum values of the angular diameter distances in the general cosmic models.

\begin{tabular}{ccc}
\hline Model & $\mathrm{Z}_{\max }$ & $\mathrm{d}_{\mathrm{A}_{\max }} / \mathrm{Mpc}$ \\
\hline $\mathrm{A}$ & 1.6442 & 1765.5434 \\
$\mathrm{~B}$ & 1.6254 & 1777.5844 \\
$\mathrm{C}$ & 1.6467 & 1773.6561 \\
$\mathrm{D}$ & 1.6118 & 1780.2004 \\
$\mathrm{E}$ & 1.5778 & 1777.4298 \\
\hline
\end{tabular}




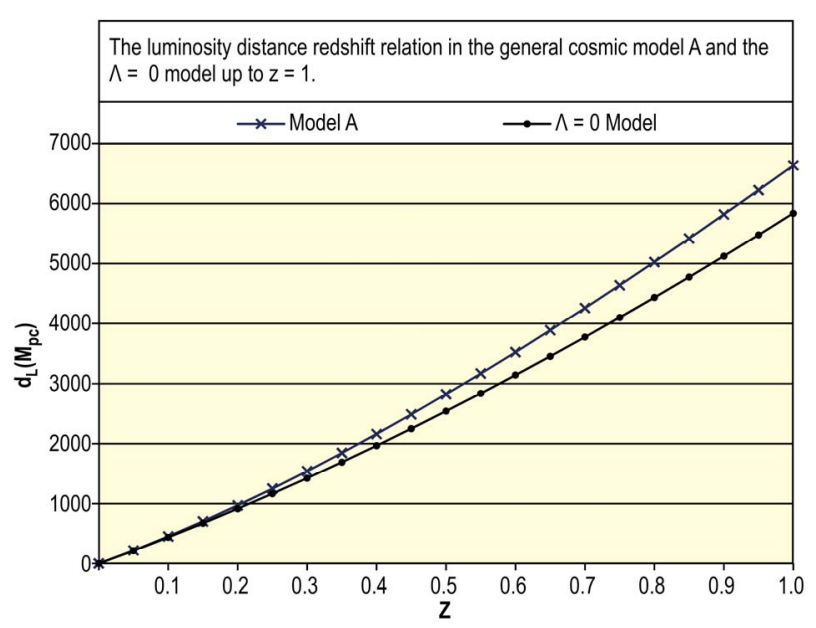

(a)

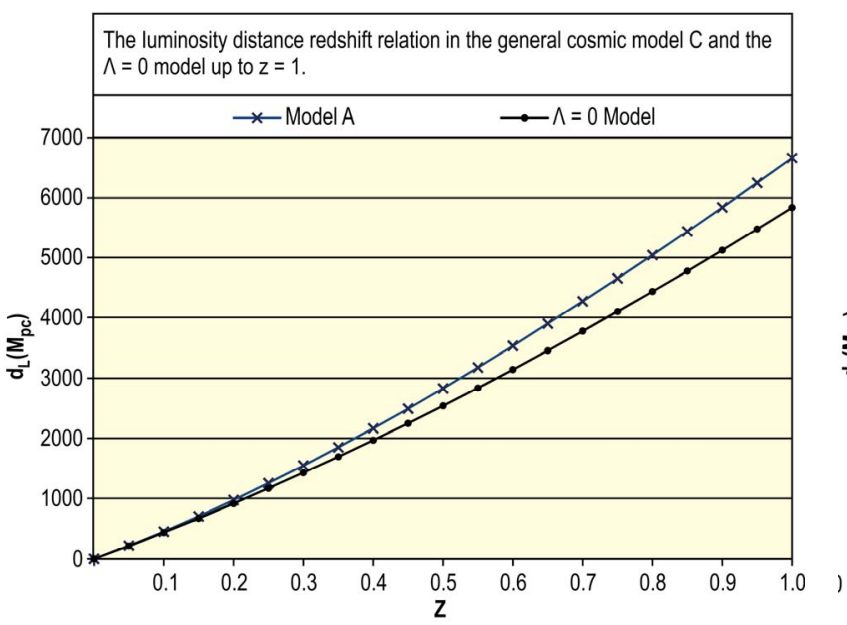

(c)

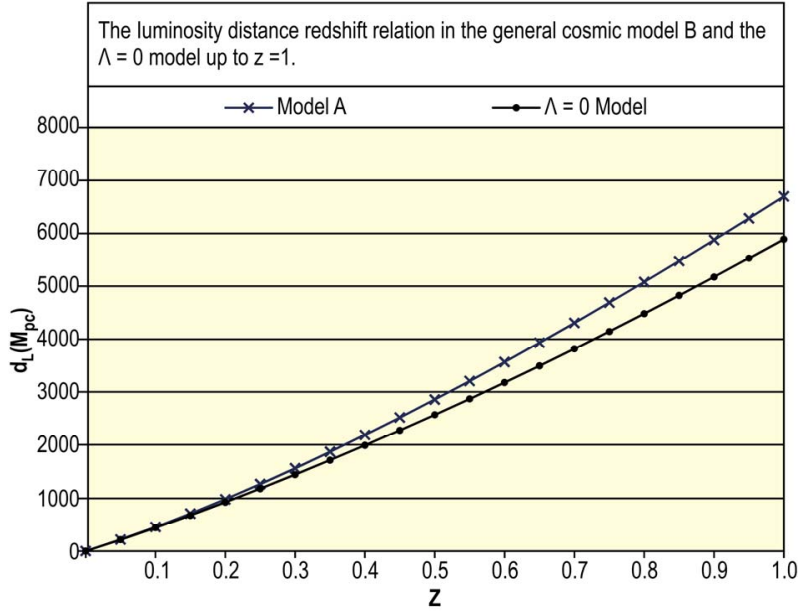

(b)

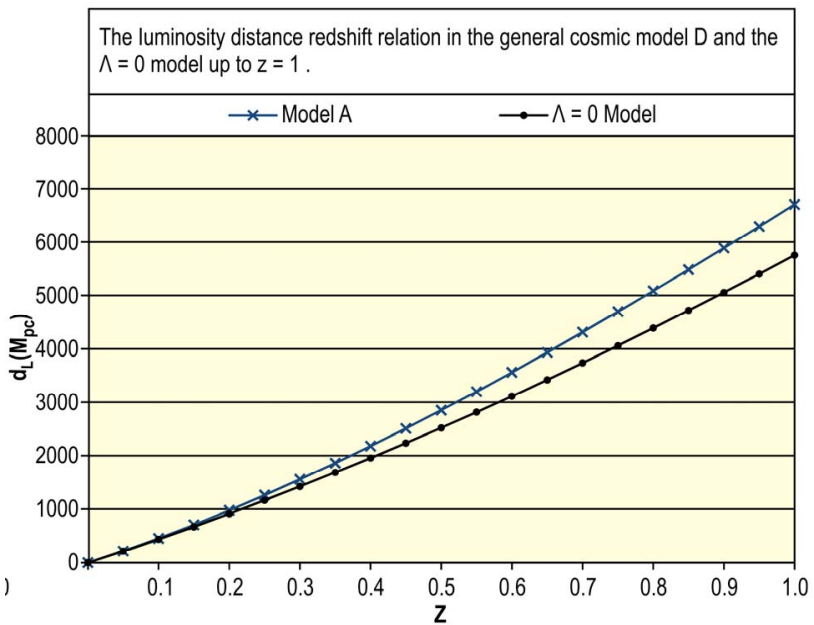

(d)

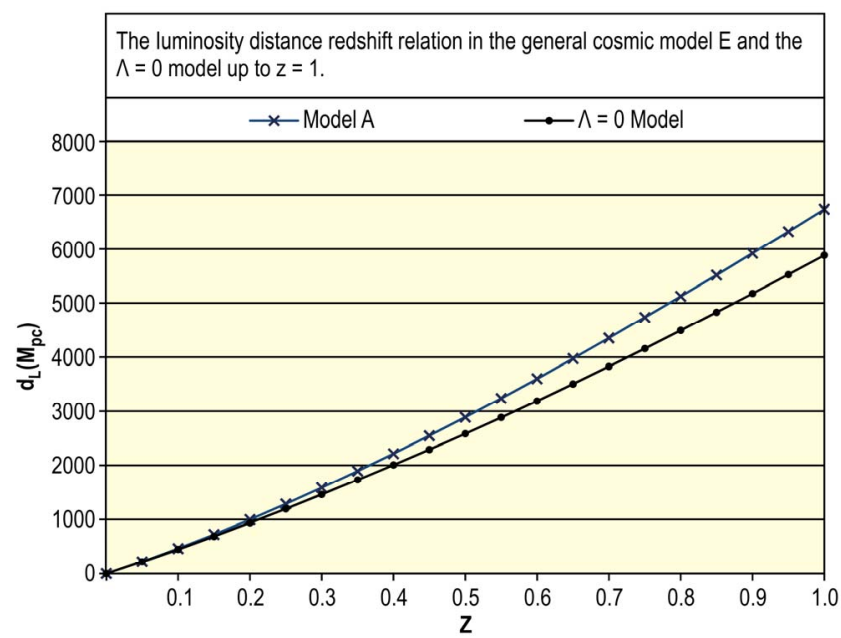

(e)

Figure 2. (a) The luminosity distance redshift relation in the general cosmic model $A$ and $\Lambda=0$ model up to $z=1 ;$ (b) The luminosity distance redshift relation in the general cosmic model $B$ and $\Lambda=0$ model up to $z=1$; (c) The luminosity distance redshift relation in the general cosmic model $C$ and $\Lambda=0$ model up to $z=1$; (d) The luminosity distance redshift relation in the general cosmic model $D$ and $\Lambda=0$ model up to $z=1$; (e) The luminosity distance redshift relation in the general cosmic $\operatorname{model} \mathrm{E}$ and $\Lambda=0$ model up to $z=1$. 
distributions in the general models up to $Z=1$ exhibited in Figure 4. It is prominent that $m-M$ increases continuously with $z$ in all general models, and the distributions of these models coincide on each other.

Values of $d_{h}\left(t_{0}\right), M_{h}\left(t_{0}\right), M_{m}\left(t_{0}\right), N_{M W}$ and $N_{\text {COMA }}$ were computed in the general models at the present time using Equations (11) and (22)-(25) respectively. These values and their possible ranges are shown in Table 2. More details about calculations of these estimations are given in the next section.

\section{Accuracy and Errors}

In computing the proper distance $d_{p}\left(t_{0}\right)$ of an extragalactic object at the present time in terms of its redshift $z$ one can get an average value of $d_{p}\left(t_{0}\right)$ to good degree of accuracy by applying Equation (7), where the parameters $H_{0}, \Omega_{m, 0}$ and $\Omega_{\Lambda, 0}$ have their average values in the general model $A$ obtained from [1].

Nevertheless, more accurate value of $d_{p}\left(t_{0}\right)$ can be obtained by calculating $d_{p}\left(t_{0}\right)$ in all the possible observed 163 models of the case A [1]. Assume the average of these 163 values of $d_{p}\left(t_{0}\right)$ is $\left\langle d_{p}\left(t_{0}\right)\right\rangle$, and the maximum and minimum values of $d_{p}\left(t_{0}\right)$ are $d_{p_{\max }}\left(t_{0}\right), d_{p_{\min }}\left(t_{0}\right)$ respectively. Let

$D_{1}=d_{p_{\max }}\left(t_{0}\right)-\left\langle d_{p}\left(t_{0}\right)\right\rangle$,

$D_{2}=\left\langle d_{p}\left(t_{0}\right)\right\rangle-d_{p_{\min }}\left(t_{0}\right)$. Then the accurate value of the proper distance of the extragalactic object is

$$
d_{p}\left(t_{0}\right)=\left\langle d_{p}\left(t_{0}\right)\right\rangle_{-D_{2}}^{+D_{1}} \text { Mpc. }
$$

This procedure was used to determine the average estimations of the parameters $d_{h}\left(t_{0}\right), M_{h}\left(t_{0}\right), M_{m}\left(t_{0}\right), N_{M W}$ and $N_{\text {COMA }}$ with their possible ranges in the general models as illustrated in Table 2.

\section{Conclusions}

In this paper distributions of the proper distance, luminosity distance, angular diameter distance and distance modulus were investigated in terms of the redshift of an

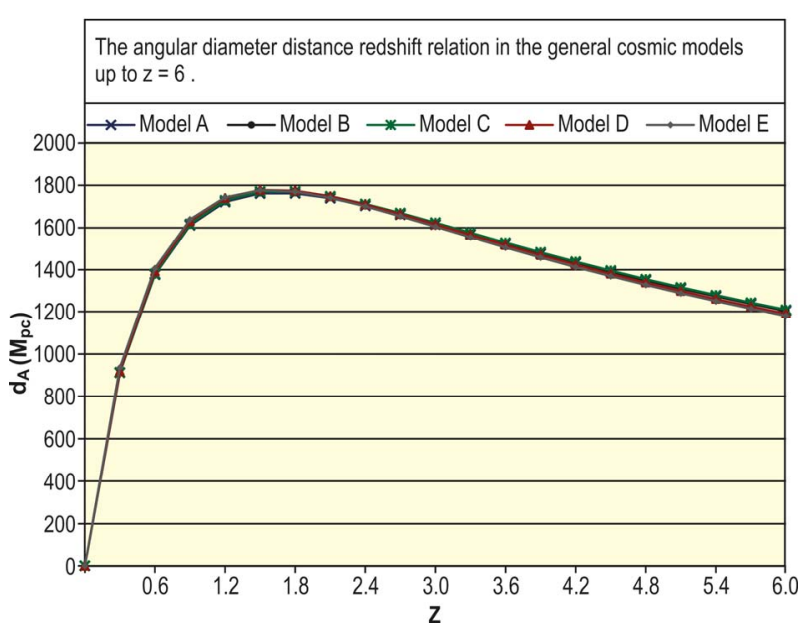

Figure 3. The angular diameter distance redshift relation in the general cosmic models up to $z=6$.

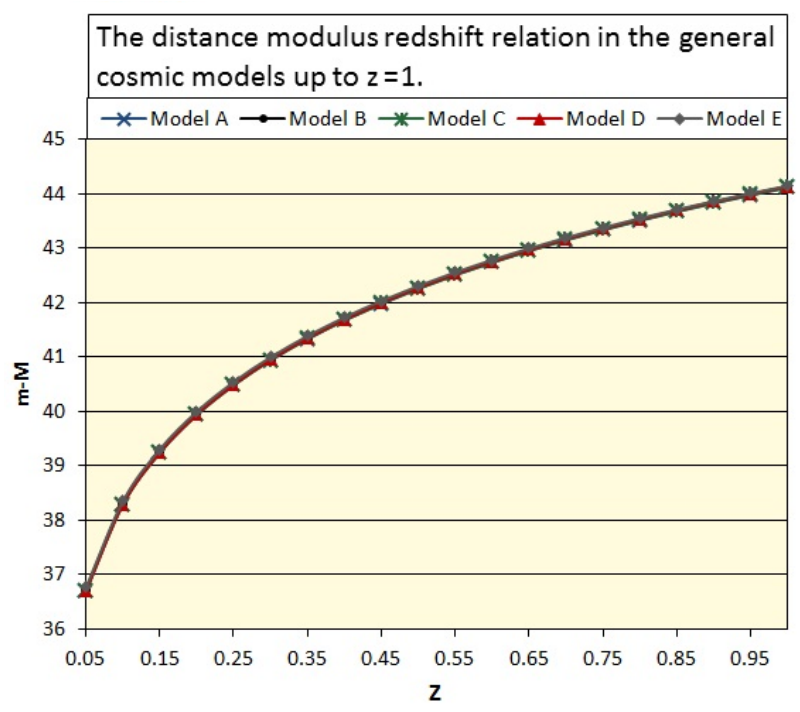

Figure 4. The distance modulus redshift relation in the general cosmic models up to $z=6$.

Table 2. Estimation of the horizon distance, the total mass and the mass of matter within the horizon distance, the equivalent numbers of the Milky way-like galaxies and the Coma-like clusters to the mass of matter in the general cosmic models at the present time.

\begin{tabular}{|c|c|c|c|c|c|}
\hline Model & $d_{h}\left(t_{0}\right) / \mathrm{Gpc}$ & $M_{h}\left(t_{0}\right) / 10^{22} M_{\odot}$ & $M_{m}\left(t_{0}\right) / 10^{22} M_{\odot}$ & $N_{M W} / 10^{9}$ & $N_{\text {СОМА }} / 10^{6}$ \\
\hline A & $14.2969_{-0.4730}^{+0.5320}$ & $341.9792_{-45.5438}^{+56.4126}$ & $91.7192_{-5.5269}^{+5.8286}$ & $1146.5031_{-69.5031}^{+72.4969}$ & $458.6012_{-27.6012}^{+29.3988}$ \\
\hline B & $14.1588_{-0.7356}^{+0.6701}$ & $332.7138_{-45.5391}^{+65.6780}$ & $92.9571_{-6.7648}^{+5.9960}$ & $1161.9732_{-84.9732}^{+75.0268}$ & $464.8036_{-33.8036}^{+30.1964}$ \\
\hline $\mathrm{C}$ & $14.2780_{-0.6502}^{+0.6855}$ & $348.9703_{-103.0449}^{+106.6920}$ & $92.5297_{-13.2374}^{+15.9785}$ & $1156.6206_{-165.6206}^{+199.3794}$ & $462.6408_{-66.6408}^{+80.3592}$ \\
\hline $\mathrm{D}$ & $13.8666_{-1.0556}^{+1.0969}$ & $352.3645_{-106.4391}^{+102.6978}$ & $98.9397_{-19.6474}^{+15.5614}$ & $1236.7478_{-245.7478}^{+194.2522}$ & $494.6991_{-98.6991}^{+78.3009}$ \\
\hline $\mathrm{E}$ & $13.8070_{-0.9960}^{+1.2323}$ & $324.5173_{-123.0116}^{+235.7807}$ & $98.1947_{-18.9024}^{+16.3064}$ & $1227.4336_{-236.4336}^{+203.5664}$ & $490.9729_{-94.9729}^{+82.0271}$ \\
\hline
\end{tabular}


extragalactic object in the light of the five general cosmic models which were constructed in a previous paper. It is found that all of these cosmological distances increase continuously with redshift except the angular diameter distance which increases very rapidly with redshift to maximum value and then it decreases gradually with increasing $z$.

Calculations of the horizon distance, the total mass and the mass of matter within the horizon distance, the equivalent numbers of the Milky Way-like galaxies and the Coma-like clusters of galaxies to the mass of matter were all obtained in the general cosmic models at the present time.

\section{Acknowledgements}

This paper was funded by the Deanship of Scientific Re- search (DSR), King Abdulaziz University, Jeddah. The author, therefore, acknowledges with thanks DSR technical and financial support.

\section{REFERENCES}

[1] F. A. Bukhari, "Journal of King Abdulaziz University: Science," 2013.

[2] B. Ryden, "Introduction to Cosmology," Addison \& Wesley, Boston, 2003.

[3] S. Weinberg, "Gravitation and Cosmology," John Wiley \& Sons, Ltd., Hoboken, 1972.

[4] J. N. Islam, "An Introduction to Mathematical Cosmology," Cambridge University Press, Cambridge, 2002.

[5] I. Morison, "Introduction to Astronomy and Cosmology," John Wiley \& Sons, Ltd., Hoboken, 2008. 